\title{
Anti-Inflammatory Activity of Pleurotus ostreatus, a Culinary Medicinal Mushroom, in Wistar Rats
}

\author{
W. J. A. Banukie N. Jayasuriya $\mathbb{D}^{1},{ }^{1}$ Shiroma M. Handunnetti, ${ }^{2}$ Chandanie A. Wanigatunge, ${ }^{3}$ \\ Gita H. Fernando, ${ }^{3}$ D. Thusitha U. Abeytunga $\mathbb{D D}^{4}{ }^{4}$ and T. Sugandhika Suresh ${ }^{5}{ }^{5}$ \\ ${ }^{1}$ Department of Pharmacy and Pharmaceutical Sciences, Faculty of Allied Health Sciences, University of Sri Jayewardenepura, \\ Nugegoda, Sri Lanka \\ ${ }^{2}$ Institute of Biochemistry, Molecular Biology and Biotechnology, University of Colombo, Colombo, Sri Lanka \\ ${ }^{3}$ Department of Pharmacology, Faculty of Medical Sciences, University of Sri Jayewardenepura, Nugegoda, Sri Lanka \\ ${ }^{4}$ Department of Chemistry, Faculty of Science, University of Colombo, Colombo, Sri Lanka \\ ${ }^{5}$ Department of Biochemistry, Faculty of Medical Sciences, University of Sri Jayewardenepura, Nugegoda, Sri Lanka
}

Correspondence should be addressed to T. Sugandhika Suresh; sugandhika@sjp.ac.lk

Received 3 May 2019; Revised 31 October 2019; Accepted 7 February 2020; Published 5 March 2020

Academic Editor: Karin Kraft

Copyright (c) 2020 W. J. A. Banukie N. Jayasuriya et al. This is an open access article distributed under the Creative Commons Attribution License, which permits unrestricted use, distribution, and reproduction in any medium, provided the original work is properly cited.

\begin{abstract}
Context. Pleurotus ostreatus (P.o) is a culinary mushroom which is commonly called as "oyster mushroom" belonging to the Basidiomycetous fungi of the order Agaricales and family Pleurotaceae. Objectives. The present study investigates the antiinflammatory potential of P.o and the underlying mechanisms of activity. Materials and Methods. Anti-inflammatory activity was evaluated using suspensions of freeze-dried and powdered (SFDP) P.o and acetone extract (AE) of P.o in normal and alloxaninduced diabetic rats using the carrageenan-induced rat paw oedema model. The mechanisms by which P.o is mediating the antiinflammatory activity were studied using in vivo and in vitro assays. Results. At doses of 500-1000 mg/kg, the SFDP of P.o showed long-lasting activity at both early and late phases of carrageenan-induced rat paw oedema. The dose of $750 \mathrm{mg} / \mathrm{kg}$ showed the most potent inhibitory activity (92\% inhibition) in healthy rats. The AE of P.o showed maximum inhibition of oedema of $87 \%$. P.o exerted protective effects on the inflammatory pathologies in rats with diabetes. The possible mechanisms by which P.o mediates the anti-inflammatory activity were antihistamine activity (52.1\%), inhibition of cell migration to the site of inflammation (45.4\%), in vitro membrane stabilizing activity $(52.6 \%)$, and inhibition of nitric oxide (NO) production $(91.2 \%)(P<0.05)$. Dose-dependent inhibition of NO production was seen with in vitro treatment of rat peritoneal cells with AE of P.o $(r=0.95 ; P<0.05)$. Discussion and Conclusion. The promising activity of culinary mushroom P.o against inflammation suggests its potential application as a functional food during inflammatory conditions.
\end{abstract}

\section{Introduction}

Inflammation is a protective response to tissue injury which usually subsides after healing occurs [1]. Inflammation can be triggered by an innocuous agent or by an autoimmune process, as in rheumatoid arthritis [1]. During the process of inflammation, proinflammatory cells (macrophages and monocytes) release proinflammatory mediators, such as tumour necrosis factor- $\alpha$ (TNF- $\alpha$ ), cytokines (interleukin (IL)-1 and IL-2), prostaglandin $\mathrm{E}_{2}$, and nitric oxide (NO) [2].
Increased expression and activity of cyclooxygenase (COX)-2 and inducible nitric oxide synthase (iNOS) also play an important role in the inflammatory process [3]. In such cases, the defense reactions may cause tissue injury where anti-inflammatory drugs may be needed to overcome the inflammatory process. Currently available anti-inflammatory drugs possess side effects which can cause problems during clinical use [4]. Nonsteroidal anti-inflammatory drugs (NSAIDs) which are COX-2 inhibitors are known to cause serious gastrointestinal disturbances including 
bleeding, ulceration, and other effects such as hypersensitivity reactions [2]. This has led to an increase in demand for natural products with anti-inflammatory activity with fewer side effects. Plants as well as mushrooms have been targeted in the search for new anti-inflammatory agents [5]. Some plants and mushrooms have been screened and identified as sources to treat inflammation $[6,7]$.

Pleurotus species which are commonly called "oyster mushrooms" belong to the Basidiomycetes fungi of the order Agaricales and family Pleurotaceae. They are characterized by a fruiting body with an eccentric stalk and a wide cap [8]. Pleurotus ostreatus is an edible mushroom commonly known as American oyster.

Studies have reported various properties of P.o such as antinociceptive [9], hypocholesterolaemic [10], antioxidant [11], and antitumour effects [12]. Our recent investigations also showed the oral hypoglycaemic property of P.o and its probable mechanism of action $[13,14]$. We have also demonstrated the value of P.o as a functional food in diabetes mellitus [14].

Previous studies have reported [15] anti-inflammatory effects of P. eous [5], P. pulmonarius [6], P. sajor-caju [16], P. florida [17], P. eryngii [18], and $P$. citrinopileatus [19]. Bobek and Galbavy [20] studied the anti-inflammatory activity of $P . o$ in an acute colitis-induced rat model while Rivero-Pérez et al. [21] evaluated the anti-inflammatory activity of P.o in mouse ears treated with 12-O-tetradecanoylphorbol-13acetate. Jedinak et al. [22] reported the anti-inflammatory mechanisms of P.o using lipopolysaccharide-stimulated RAW264.7 macrophages. However, anti-inflammatory properties and underlying molecular mechanisms of P.o using a carrageenan-stimulated inflammatory model have not been addressed.

Therefore, the purpose of this study was to investigate the anti-inflammatory potential of P.o using carrageenaninduced rat paw oedema model and to identify the possible mechanisms underlying the activity. Effectiveness of P.o in the treatment of inflammatory pathologies in diabetic rats was also evaluated for the first time.

\section{Methods}

2.1. Study Setting. The study was conducted at the Animal House and Department of Biochemistry, Faculty of Medical Sciences, University of Sri Jayewardenepura, Sri Lanka, and the Institute of Biochemistry, Molecular Biology and Biotechnology, University of Colombo, Sri Lanka. Ethical clearance was granted by the Ethics Review Committee of the Faculty of Medical Sciences, University of Sri Jayewardenepura, Sri Lanka (no. 380/8).

2.2. Experimental Animals. Healthy adult Wistar rats (200-250 g) were purchased from the Medical Research Institute Colombo. They were housed under standardized animal house conditions and had access to food (WHO recommended food formula: maize $40.1 \mathrm{~kg}$, broken brown rice $10 \mathrm{~kg}$, rice bran $2.5 \mathrm{~kg}$, wheat bran $2 \mathrm{~kg}$, wheat flour $13.5 \mathrm{~kg}$, fish meal $8 \mathrm{~kg}$, soya meal $8 \mathrm{~kg}$, sugar $2.5 \mathrm{~kg}$, soya oil $2 \mathrm{~kg}$, grass powder $3 \mathrm{~kg}$, bone meal $1.5 \mathrm{~kg}$, mineral mix $0.4 \mathrm{~kg}$, vitamin mix $0.24 \mathrm{~kg}, \mathrm{NaCl} 0.2 \mathrm{~kg}$, beta mix E50 $0.02 \mathrm{~kg}, \mathrm{DL}$ methionine $0.05 \mathrm{~kg}$, milk powder 6 spoons, and vitamin $\mathrm{B}$ complex 600 tablets $/ 100 \mathrm{~kg}$ ) and water ad libitum.

2.3. Collection and Preparation of Mushrooms. Fresh P.o grown using the spawn provided by the Mushroom Cultivation Centre, Export Research Board (Ratmalana, Sri Lanka), were collected from a local farm. The identification and authentication were done by studying the spore print and the shape of the cap (fan-shaped) and the stipe (eccentric). Fresh P.o (1 kg) was washed with water to remove soil particles, freeze-dried (Eyela, FD-5N, Japan), and ground with a commercial blender (Sonica, SA-317, China). The SFDP P.o were freshly prepared with distilled water (DW) prior to the feeding of rats.

2.4. Extraction of Mushroom. Fresh mushroom (3.0 kg) was homogenized with a homogenizer (Ultra-Turrax ${ }^{\circledR}$, T 25 basic, IKA-Werke, Germany) and left overnight soaked in $1.5 \mathrm{~L}$ of distilled acetone. The solution was removed and again extracted using $1.5 \mathrm{~L}$ of acetone according to the same method stated. The solution was filtered using a filter paper to remove particles. The extract was concentrated using a rotary evaporator (Eyela, N-N series, Japan) and freeze-dried (FD-5N, Eyela, Japan). The dried material (65 g) was stored in a refrigerator. The AE of P.o was dissolved in DW by sonication prior to feeding to rats.

\subsection{Anti-Inflammatory Activity}

2.5.1. Anti-Inflammatory Activity of P. ostreatus in Carrageenan-Induced Paw Oedema in Healthy Wistar Rats. The anti-inflammatory activity of P.o was determined using the carrageenan-induced paw oedema model [23]. Healthy, male, Wistar rats $(n=48)$ were selected and the volume of the left hind paw of each rat was measured using a plethysmometer $\left(V_{\mathrm{o}}\right)$ (Panlab s.I., Barcelona, Spain). These rats were randomly divided into eight equal groups and treated orally in the following manner. Groups 1, 2, 3, 4, and 5 were orally administered with five different doses of SFDP P.o, $125 \mathrm{mg} / \mathrm{kg}, 250 \mathrm{mg} / \mathrm{kg}, 500 \mathrm{mg} / \mathrm{kg}, 750 \mathrm{mg} / \mathrm{kg}$, and $1000 \mathrm{mg} /$ $\mathrm{kg}$, respectively. Group 6 was given AE of P.o at a dose of $500 \mathrm{mg} / \mathrm{kg}$. Group 7 was treated with the reference drug, indomethacin $(10 \mathrm{mg} / \mathrm{kg})$, whereas rats in group 8 received $2.5 \mathrm{~mL}$ of distilled water and served as the control group. After 1 hour, $0.1 \mathrm{~mL}$ of $1 \%$ carrageenan suspension in normal sterile saline was injected subcutaneously into the plantar surface of the left hind paw of rats under mild ether anaesthesia. The left hind paw volumes of these rats were measured at hourly intervals up to $5^{\text {th }}$ hour $\left(V_{\mathrm{t}}\right)$. Using these volumes, increase in paw oedema and percent inhibition of paw oedema were calculated as follows:

$\%$ inhibition of oedema $=\frac{\left(V_{\mathrm{t}}-V_{0}\right)-\left(V_{\mathrm{t}}-V_{0}\right)_{\text {treated }} \times 100}{\left(V_{\mathrm{t}}-V_{0}\right)_{\text {control }}}$. 
2.5.2. Anti-Inflammatory Activity of P. ostreatus in Carrageenan-Induced Paw Oedema in Alloxan-Induced, Diabetic Wistar Rats. The rats were injected with alloxan monohydrate dissolved in normal sterile saline at a dose of $40 \mathrm{mg} /$ $\mathrm{kg}$ intravenously to induce diabetes. After 72 hours, the rats $(n=30)$ showing fasting serum glucose levels above $180 \mathrm{mg} /$ $\mathrm{dL}$ were selected for the study. Anti-inflammatory activity of SFDP P.o (doses used: $500 \mathrm{mg} / \mathrm{kg}$ and $1000 \mathrm{mg} / \mathrm{kg}$ ), AE of P.o $(500 \mathrm{mg} / \mathrm{kg})$, and indomethacin $(10 \mathrm{mg} / \mathrm{kg})$ were determined according to the method described above. The group receiving $2.5 \mathrm{~mL}$ of DW served as the control group. The mid- and high doses of SFDP P.o, $500 \mathrm{mg} / \mathrm{kg}$ and $1000 \mathrm{mg} / \mathrm{kg}$, were selected from the study conducted with healthy rats.

\subsection{Mechanisms of Anti-Inflammatory Activity}

2.6.1. Antihistamine Activity. Healthy, male, Wistar rats $(n=24)$ were selected, and fur on the left posterior lateral side was removed by gentle shaving. Rats were randomly assigned to three groups after 24 hours and treated orally in the following manner. Group 1: $500 \mathrm{mg} / \mathrm{kg}$ of AE of P.o; Group 2: $0.67 \mathrm{mg} / \mathrm{kg}$ of chlorpheniramine; and Group 3: $2.5 \mathrm{~mL}$ of DW. After 1 hour, $50 \mu \mathrm{L}$ of $200 \mu \mathrm{g} / \mathrm{mL}$ of histamine dihydrochloride in normal sterile saline was subcutaneously injected into the shaved area of the skin, and the area of the wheal formed was determined after 2 minutes [24].

2.6.2. Assay for Carrageenan-Induced Infiltration of Rat Peritoneal Cells. Three groups of rats $(n=18)$ were orally treated with $500 \mathrm{mg} / \mathrm{kg}$ of AE of P.o, $10 \mathrm{mg} / \mathrm{kg}$ of prednisolone, and DW. After 1 hour, $1 \mathrm{mg} / \mathrm{mL}$ of carrageenan in normal sterile saline was injected intraperitoneally at a dose of $5 \mathrm{mg} / \mathrm{kg}$ under ether anaesthesia. After 2 hours, $40 \mathrm{~mL}$ of sterile phosphate-buffered saline (PBS) with a $\mathrm{pH}$ of 7.4 was injected into the peritoneal cavity. Five minutes later, $35 \mathrm{~mL}$ of peritoneal fluid was drained using an $18 \mathrm{G}$ cannula and centrifuged at $150 \mathrm{~g}$ for 10 minutes at $4^{\circ} \mathrm{C}$. The peritoneal cells were resuspended in $1 \mathrm{~mL}$ of PBS after removing the supernatant. A $50 \mu \mathrm{L}$ cell suspension was mixed with $10 \mu \mathrm{L}$ of $1 \%$ neutral red. Phagocytic/macrophage cell counts were made using a haemocytometer [24].

2.6.3. In Vivo Assay for Nitric Oxide Production by Peritoneal Cells. Three groups of rats $(n=18)$ were treated with $500 \mathrm{mg} / \mathrm{kg}$ of AE of P.o, $10 \mathrm{mg} / \mathrm{kg}$ of prednisolone, and DW. Peritoneal cells exposed to extracts in vivo were harvested from rats orally treated with extract as described above. The in vivo NO inhibitory effect of the AE of P.o and prednisolone was studied according to the method described by Handunnetti et al. [24]. The peritoneal cells collected from each rat were plated in 96-well tissue culture plates at $1 \times 10^{6}$ cells/mL in RPMI 1640 medium (GIBCO BRL, Life Technologies, Scotland) supplemented with $1 \%$ bovine serum albumin (BSA) (Sigma Chemicals Company, St Louis, Mo, USA) and incubated at $37^{\circ} \mathrm{C}$ in $5 \% \mathrm{CO}_{2}$ incubator (Sanyo Electric. Co. Ltd., Osaka, Japan). The culture supernatant was aspirated from each well, after 24 hours and then centrifuged at $10000 \mathrm{~g}$ for 10 minutes and the clear supernatant was obtained. In order to measure the NO concentration, $100 \mu \mathrm{L}$ of culture supernatant was mixed with an equal volume of Griess reagent (mixture of equal proportion of $1 \%$ sulphanilamide in 5\% phosphoric acid and $0.1 \% \mathrm{n}-(1-$ naphthyl) ethylenediamine hydrochloride in DW) and incubated at $25^{\circ} \mathrm{C}$ for 15 minutes and optical density (OD) was read at $540 \mathrm{~nm}$ in an ELISA plate reader (ELX 800, Bio-Tek Instruments Inc, USA). The NO concentration was calculated using a calibration curve between 0.195 and $100 \mu \mathrm{M}$ $\mathrm{NaNO}_{2}$.

2.6.4. In Vitro Assay for Nitric Oxide Production by Peritoneal Cells. The in vitro NO inhibitory effect was studied according to the method described by Maccioni et al. [25]. The viability of peritoneal cells after 30 minutes of incubation with different concentrations of AE of P.o was assessed by Trypan blue exclusion test [24]. Peritoneal cells harvested from rats administered with carrageenan $(5 \mathrm{mg} /$ $\mathrm{kg}$ ) intraperitoneally were placed in 96-well tissue culture plate. The cells were treated in vitro with $3.90-125 \mu \mathrm{g} / \mathrm{mL}$ of AE of P.o in RPMI 1640 medium supplemented with $1 \%$ $\mathrm{BSA}$ at $37^{\circ} \mathrm{C}$ in a $5 \% \mathrm{CO}_{2}$ incubator for 30 minutes. In addition, peritoneal cells were treated with RPMI 1640 medium as a negative control. Cells were centrifuged at $150 \mathrm{~g}$ for 2 minutes and resuspended in culture medium containing 1\% BSA and cultured for 24 hours after which the culture supernatant was aspirated. The culture supernatant was aspirated from each well, after 24 hours. It was then centrifuged at $10000 \mathrm{~g}$ for 10 minutes, to obtain a clear supernatant, and the NO concentration was measured.

2.6.5. Assay for Membrane Stabilizing Activity. This assay was performed using heat-induced haemolysis of rat erythrocytes. A ten-fold dilution series ranging from 0.001 to $1000 \mu \mathrm{g} / \mathrm{mL}$ of AE of P.o and aspirin ( $n=6 \mathrm{each}$ ) was made using PBS. PBS was used as a control. Vials containing $20 \mu \mathrm{L}$ of rat blood and different concentrations of $\mathrm{AE}$ of P.o, aspirin, and PBS ( $1 \mathrm{~mL}$ each) were prepared in triplicate. The vials were incubated at $37^{\circ} \mathrm{C}$ for 15 minutes and centrifuged at $1500 \mathrm{~g}$ for 3 minutes. The supernatants were removed and the cells were resuspended in $1 \mathrm{~mL}$ of PBS. The vials were then incubated at $54^{\circ} \mathrm{C}$ for 25 minutes, centrifuged at $1500 \mathrm{~g}$ for 5 minutes, and supernatants were obtained $(200 \mu \mathrm{L})$; OD value was measured at $540 \mathrm{~nm}$. Percentage inhibition of haemolysis was calculated with respect to the controls:

percent inhibition of haemolysis $=\left[\frac{(\mathrm{OD})_{\text {control }}-(\mathrm{OD})_{\text {sample }}}{(\mathrm{OD})_{\text {control }}}\right] \times 100$.

2.7. Statistical Analysis. Results were presented as mean \pm SEM. The results were analyzed for statistical significance using Student's $t$-test. Effects of different doses were analyzed by analysis of variance (ANOVA). Statistical analysis was done 
using Microsoft Excel 2007 version and SPSS 17. $P$ values $<0.05$ were considered as significant.

\section{Results}

3.1. Anti-Inflammatory Activity of P. ostreatus in Carrageenan-Induced Paw Oedema in Healthy Wistar Rats. The results of anti-inflammatory activity of P.o in healthy Wistar rats are shown in Figure 1. When compared to the control group, treatment with SFDP P.o at doses of $250-1000 \mathrm{mg} / \mathrm{kg}$ and indomethacin showed significant $(P<0.05)$ inhibition of rat paw oedema. The low dose $(125 \mathrm{mg} / \mathrm{kg})$ did not significantly $(P>0.05)$ impair paw oedema at $2-5$ hours whereas the reference drug indomethacin showed significant inhibition up to the $5^{\text {th }}$ hour. The SFDP P.o at doses of $500-1000 \mathrm{mg} / \mathrm{kg}$ showed long-lasting activity at both early and late phases. The SFDP P.o at $750 \mathrm{mg} / \mathrm{kg}$ showed maximum inhibition of oedema of $92 \%$ (after 5 hours) $(P<0.05)$. The inhibition pattern of the highest dose of SFDP P.o $(1000 \mathrm{mg} / \mathrm{kg})$ was similar to that of indomethacin $(P<0.05)$. However, according to Figure 1, the inhibition of carrageenan-induced paw oedema by SFDP P.o was not dosedependent. The AE of P.o showed maximum inhibition of oedema of $87 \%(P<0.01)$.

3.2. Anti-Inflammatory Activity of P. ostreatus in Carrageenan-Induced Paw Oedema in Alloxan-Induced, Diabetic Wistar Rats. The results are summarized in Figure 2. Treatment with SFDP P.o at doses of $500 \mathrm{mg} / \mathrm{kg}$ (up to $5^{\text {th }}$ hour) and $1000 \mathrm{mg} / \mathrm{kg}$ (up to $4^{\text {th }}$ hour) showed significant $(P<0.05)$ inhibition of paw oedema in diabetic rats when compared to the diabetic control group. The AE of P.o $(500 \mathrm{mg} / \mathrm{kg})$ showed significant $(P<0.01)$ inhibition of paw oedema in diabetic rats after 1-5 hours. The anti-inflammatory effect exerted by the SFDP P.o at doses of $500 \mathrm{mg} / \mathrm{kg}$ and $1000 \mathrm{mg} / \mathrm{kg}$ and $\mathrm{AE}$ of P.o was comparable to indomethacin $(P>0.05)$. The SFDP P.o at doses of $500 \mathrm{mg} / \mathrm{kg}$ (after $1^{\text {st }}$ hour) and $1000 \mathrm{mg} / \mathrm{kg}$ (after $2^{\text {nd }}$ hours) showed maximum inhibition of oedema of $70 \%$ whereas the AE of P.o showed maximum inhibition of oedema of $86 \%(P<0.01)$. The dose of $1000 \mathrm{mg} / \mathrm{kg}$ showed highest activity at an early phase of carrageenan-induced paw oedema whereas the dose of $500 \mathrm{mg} / \mathrm{kg}$ showed long-lasting activity at both early and late phases. However, according to Figure 2, the inhibition of carrageenan-induced paw oedema in alloxan-induced, diabetic Wistar rats by SFDP P.o was not dose-dependent.

3.3. Antihistamine Activity. Compared to the control, treatment with AE of P.o and chlorpheniramine significantly reduced $(P<0.0001)$ the area of wheal formed $(52.1 \pm 1.1 \%$ and $57.9 \pm 1.5 \%$, respectively) on the skin by the injection of histamine. However, the antihistamine effect exerted by AE of P.o was less than the effect exerted by chlorpheniramine.

3.4. Inhibition of Carrageenan-Induced Infiltration of Rat Peritoneal Cells. The AE of P.o and prednisolone significantly inhibited the number of phagocytic cells infiltrating

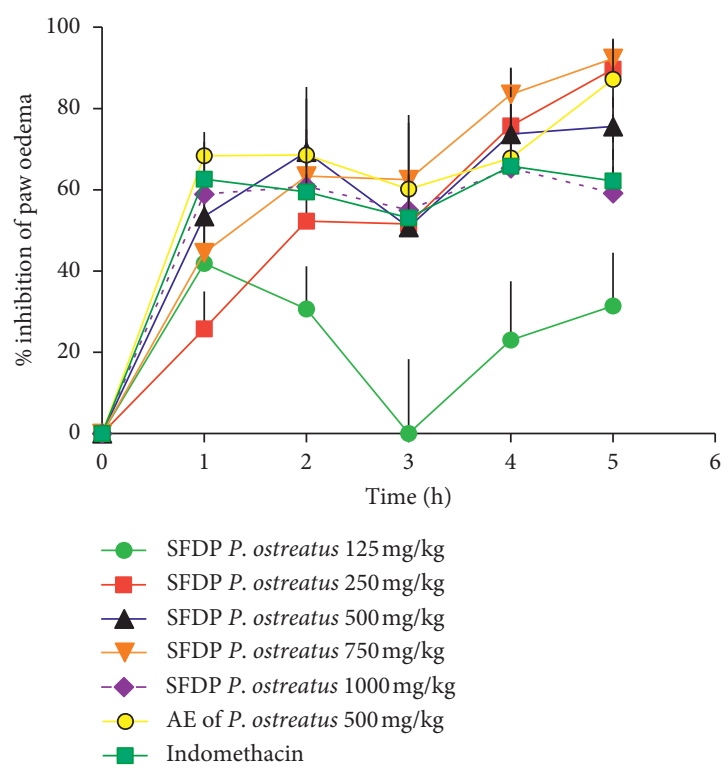

FIgURE 1: Inhibition of carrageenan-induced rat paw oedema, following oral administration of different doses of suspensions of freeze-dried and powdered $P$. ostreatus, acetone extract of $P$. ostreatus, and indomethacin using healthy rats. Values are expressed as mean \pm SEM.

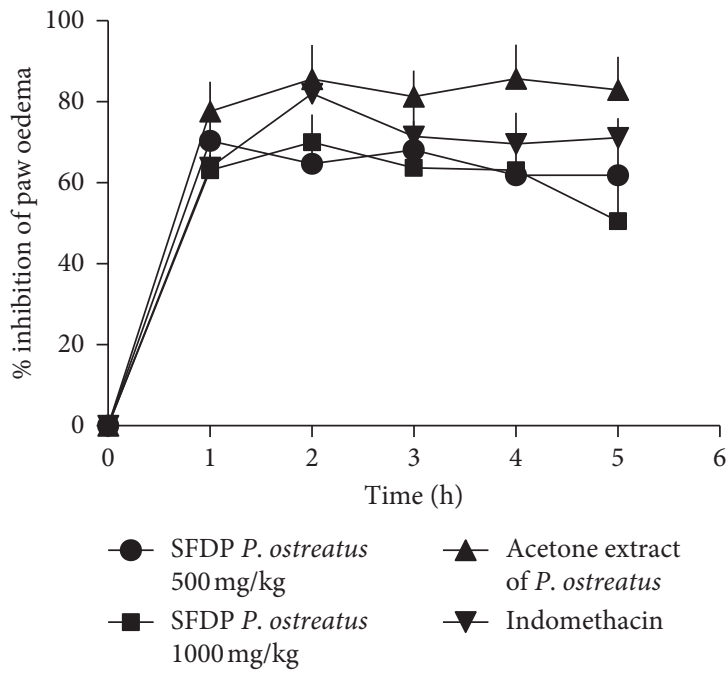

FIgURE 2: Inhibition of carrageenan-induced rat paw oedema, following oral administration of different doses of suspensions of freeze-dried and powdered $P$. ostreatus and acetone extract of $P$. ostreatus using alloxan-induced diabetic rats. Values are expressed as mean \pm SEM.

into the peritoneal cavity when compared to the control group $((P<0.01)$, (Figure 3$))$.

3.5. Inhibition of Nitric Oxide Production by Peritoneal Cells following In Vivo Treatment. Peritoneal cells harvested from rats after oral administration of $\mathrm{AE}$ of $P . o$ and prednisolone significantly inhibited NO production when compared with the control group $(91.2 \pm 1.3 \%$ and $95.6 \pm 0.2 \%$ reduction; $P<0.001$, respectively). 


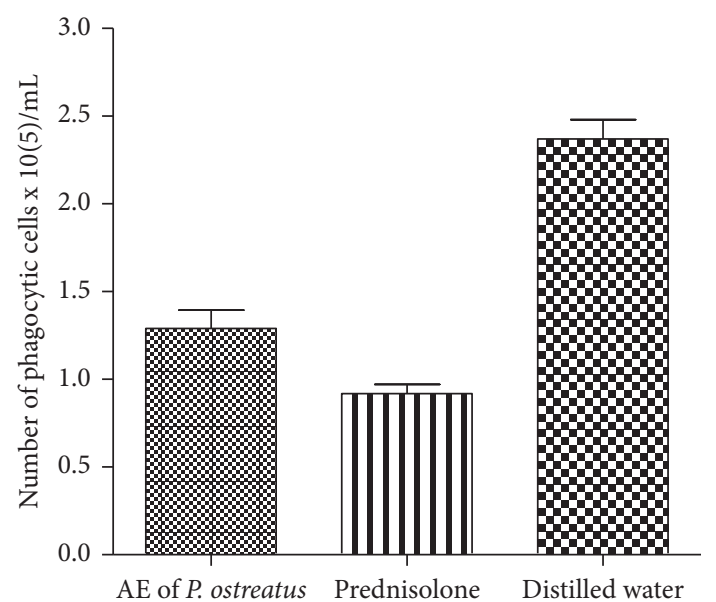

FIGURE 3: Effect of acetone extract of $P$. ostreatus on rat peritoneal cell infiltration. Values are expressed as mean \pm SEM.

3.6. Inhibition of Nitric Oxide Production by Peritoneal Cells following In Vitro Treatment. Viable cell counts obtained after 30 minutes of incubation with different concentrations of AE of P.o showed concentrations of $125 \mu \mathrm{g} / \mathrm{mL}$ (72.7\%) or lower, were not cytotoxic to peritoneal cells, and were suitable for the in vitro treatment of assay for NO inhibitory activity. Treatment of peritoneal cells with $3.90-125 \mu \mathrm{g} / \mathrm{mL}$ of $\mathrm{AE}$ of P.o in vitro significantly inhibited the NO production $(P<0.01)$. Apart from the lowest dose $(3.90 \mu \mathrm{g} / \mathrm{mL})$, other tested doses of AE of P.o demonstrated a dose-dependent inhibition of NO production $(r=0.95 ; P<0.05)$. Maximum inhibition was observed at a dose of $125 \mu \mathrm{g} / \mathrm{mL}$ $((76.9 \pm 2.9 \%)$ (Figure 4$))$.

3.7. Membrane Stabilizing Activity. Apart from the lowest concentration of $\mathrm{AE}$ of P.o $(0.001 \mu \mathrm{g} / \mathrm{mL})$, other higher concentrations significantly inhibited the heat-induced haemolysis of rat erythrocytes in vitro. The highest inhibitory activity $(52.6 \pm 2.1 \%$.) was shown by the dose of $100 \mu \mathrm{g} /$ $\mathrm{mL}$. Decrease of the inhibition of haemolysis at $1000 \mu \mathrm{g} / \mathrm{mL}$ may be due to the multicomponent nature of the AE of P.o, which could contain active compounds with different effects. Hence, it can be speculated that at the higher dose levels, anti-inflammatory activity might have been masked by the presence of the opposite effect producing active compounds. This type of dose-independent membrane stabilizing activity is reported by various studies on medicinal plants [26, 27].

With the increasing concentrations of aspirin, the inhibition of haemolysis increased dose-dependently $(r=0.76$; $P<0.05$ ) (Figure 5) according to the Pearson correlation.

\section{Discussion}

In the present study, an attempt has been made to evaluate the anti-inflammatory activity of P.o by use of the carrageenan-induced paw oedema model. Inhibition of carrageenan-induced inflammation has played a pivotal role in the development of NSAIDs and COX inhibitors [28]. The inflammatory response of carrageenan-induced paw oedema

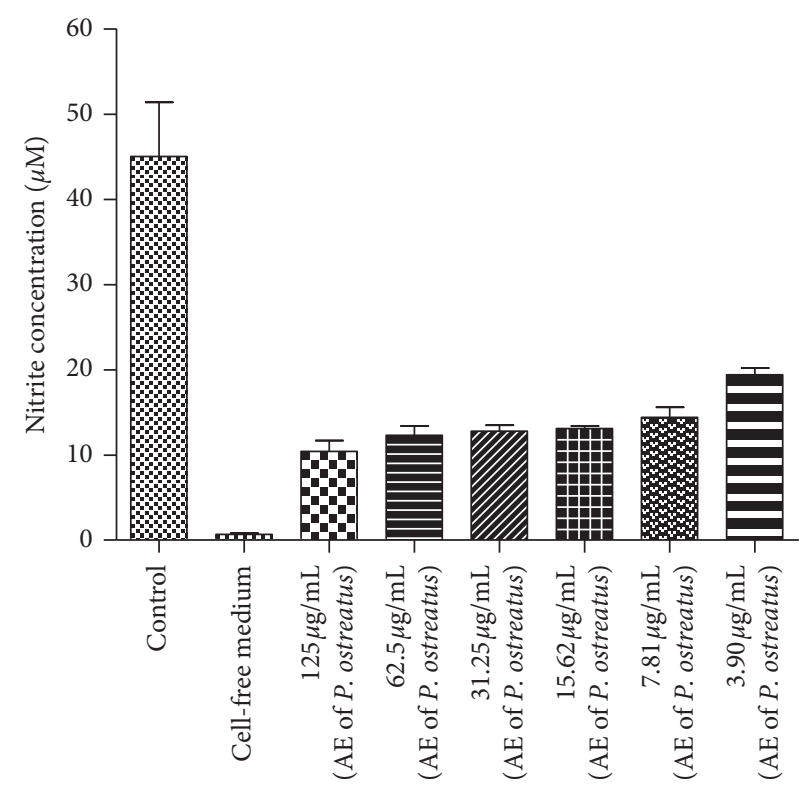

Figure 4: Effect of different concentrations of acetone extract of $P$. ostreatus on NO production by rat peritoneal cells. Values are expressed as mean \pm SEM.

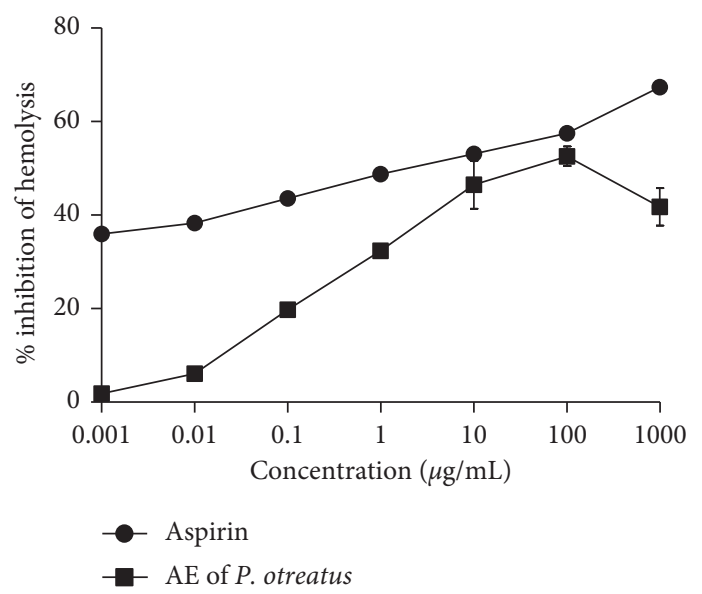

FIgURe 5: Percentage inhibition of heat-induced haemolysis of rat erythrocytes by acetone extract of $P$. ostreatus. Values are expressed as mean \pm SEM.

test is biphasic with a maintenance phase in between (2-3 hours). The initial inflammatory phase is mediated by histamine and serotonin and increase in PG synthesis at the damaged tissue and lasting up to 2 hours, whereas the delayed inflammatory phase is mediated by leukotrienes, phagocytic cells, polymorphonuclear cells, monocytes, macrophages, PGs produced by tissue macrophages, oxygen free radicals, and NO and observed from 3 to 5 hours. During the maintenance phase, kinin-like substances, such as bradykinin are predominant [28].

Oral administration of a single dose of SFDP P.o and AE of P.o exerted significant anti-inflammatory activity in normal rats. When considering the dose study (Figure 1), it was noted that the SFDP P.o at doses of $250-1000 \mathrm{mg} / \mathrm{kg}$ had exerted a significant inhibition of rat paw oedema. However, 
the inhibition of carrageenan-induced paw oedema by SFDP P.o was not dose-dependent. This is probably due to the multicomponent nature of the P.o suspensions, which could contain active compounds with different effects. Hence, it can be speculated that at the higher dose levels, anti-inflammatory activity might have been masked by the presence of the opposite effect producing active compounds. This type of dose-independent anti-inflammatory activity is reported by various studies on medicinal plants [29] and the drug tacrolimus [30].

The AE of P.o $(500 \mathrm{mg} / \mathrm{kg})$ showed maximum inhibition of $87 \%(P<0.01)$, at late phase of inflammation. Hence, the anti-inflammatory activity-guided fractionation of AE of P.o could be beneficial in the search of anti-inflammatory active agents.

The role of inflammation in diabetes has been extensively studied [31]. It has been reported that there is an increase in levels of cytokines in diabetic conditions [32]. Furthermore, diabetes results in poor anti-inflammatory capabilities [33]. Even though the efficacy of plants for the treatment of inflammatory conditions seen with diabetes has been reported, [34] studies using mushrooms are not documented.

Treatment with SFDP P.o at doses of $500 \mathrm{mg} / \mathrm{kg}$ and $1000 \mathrm{mg} / \mathrm{kg}$ and $\mathrm{AE}$ of P.o showed significant $(P<0.05)$ inhibition of paw oedema in diabetic rats which was comparable with indomethacin. Furthermore, the anti-inflammatory activity in diabetic rats, at doses of $500 \mathrm{mg} / \mathrm{kg}$ and $1000 \mathrm{mg} / \mathrm{kg}$ of SFDP P.o, and in AE of P.o (500 mg/kg) was higher than in healthy rats at early phase of carrageenaninduced paw oedema. Since the diabetes results in poor antiinflammatory capabilities, this study supports the capability of P.o in the treatment of inflammatory pathologies in rats with diabetes. Similar effect was reported by Hassanabad et al. [34]. In the present study, carrageenan-induced paw oedema in the diabetic control group was higher than in healthy control rats after 2-5 hours. Similar to the observation by Hassanabad and coworkers [34], in our study too, there was a significant increase in inflammation of diabetic mice in the control group when compared with normal mice.

The SFDP P.o and AE of P.o impaired both the initial and late phases of carrageenan-induced inflammation. This study exhibited marked antihistamine activity of AE of P.o $(52.1 \%$ at the dose of $500 \mathrm{mg} / \mathrm{kg})$, and Vasudewa et al. [9] reported the antihistamine activity of SFDP P.o $(50.7 \%$ at the dose of $1000 \mathrm{mg} / \mathrm{kg}$ ) when evaluated by the wheal test. Histamine released from mast cells increases vascular permeability which is one of the essential features of the acute inflammatory response [1]. Therefore, initial phase inhibition could be partly due to the impairment of microvascular leakage by antihistamine activity.

Cyclooxygenase is the enzyme that catalyzes the first two steps in the biosynthesis of the PGs from the substrate arachidonic acid [2]. There are two isoforms of this enzyme, COX-1 and COX-2. Different classes of compounds are known to produce anti-inflammatory activity through the inhibition of COX-2 and/or iNOS activity and/or expression $[3,35]$. COX inhibitory compounds have been isolated from mushrooms such as Grifola frondosa [36]. Glycoprotein isolated from P. citrinopileatus and water extract of P.o showed inhibition of LPS-induced production of PGE2 and $\mathrm{NO}$ through the downregulation of expression of COX-2 and iNOS in macrophages, respectively $[19,22]$. Therefore, in the present study inhibition of the late phase of carrageenan-induced paw oedema by P.o may be attributed to COX inhibition and prostaglandin synthesis. Furthermore, P.o showed marked and dose-dependent antioxidant activity as observed by Vasudewa et al. [9], which can be linked to its anti-inflammatory action in the late phase.

Phagocytic cell migration to the site of inflammation is well known [1]. Nacife et al. [37] reported carrageenaninduced leukocyte migration. This model has been used to demonstrate the inhibition of peritoneal cell migration by Ixora coccinea [24]. This model in turn can be used to study the cell migration process and the inhibitory effects of drugs on cell migration. In the present study, the AE of P.o significantly reduced the migration of phagocytic cells in response to carrageenan-induced inflammatory stimulus. Hence, in vivo peritoneal infiltration assay emphasized another mechanism responsible for anti-inflammatory activity of P.o.

Nitric oxide is produced in cells constitutively or induced by various cell activators through the oxidation of L-arginine by isoenzymes (nitric oxide synthases). Expression of iNOS, one of the isoforms, generates higher levels of NO which contributes to the immune defense [3].

The NO generated diffuses to the vascular smooth muscle, activates soluble guanylate cyclase, leading to increased intracellular cGMP levels, and promotes relaxation of the smooth muscle causing vasodilatation [3]. Increased permeability of blood vessels leads to the exudation of plasma proteins and fluids into the tissues forming oedema [38].

In this study, treatment with $\mathrm{AE}$ of P.o in vitro and in vivo significantly inhibited $\mathrm{NO}$ production by rat peritoneal cells. The results of in vitro assay show that carrageenan induced an increase in NO production in peritoneal cells which was significantly $(P<0.01)$ reduced when treated with different concentrations of AE of P.o. This study suggests that AE of P.o is an effective inhibitor against carrageenaninduced NO production. Hence, inhibition of NO is likely to be another mechanism via P.o-induced anti-inflammatory activity in the late phase of the carrageenan-induced paw oedema test. Inhibition of NO production has been observed in other Pleurotus species as well. Chen et al. [19] reported the ability of $P$. citrinopileatus in inhibiting lipopolysaccharide-induced NO production in vitro.

Moreover, apart from the lowest dose $(3.90 \mu \mathrm{g} / \mathrm{mL})$, other tested doses of AE of P.o demonstrated a dose-dependent inhibition of NO production in the present study. This suggests that the concentration of active constituents which are responsible for reducing $\mathrm{NO}$ production in $\mathrm{AE}$ of $P . o$ is increased when increasing the dose. Methanol extract of C. pruinosa also inhibited NO production in LPS-stimulated RAW264.7 cells in a dose-dependent manner [39].

In addition, AE of P.o showed membrane stabilizing activity in the heat-induced haemolysis of rat erythrocytes in vitro. On exposure to hypotonic medium, heat, and 
phenylhydrazine may cause the lysis of the red blood cell (RBC) membrane which leads to haemolysis and oxidation of haemoglobin [40]. Red blood cell membranes are similar to lysosomal membrane components [7]. Thus, the inhibition of heat-induced $\mathrm{RBC}$ membrane lysis was considered as one of the mechanisms of anti-inflammatory activity of P.o. The AE of $P$.o may stabilize the lysosomal membrane, preventing the release of inflammatory mediators and lytic enzymes. Compounds which demonstrate membrane stabilization activity inhibit early phase of inflammatory reactions by preventing the release of phospholipases [41]. Hence, the membrane stabilization potential of $\mathrm{AE}$ of P.o can be attributed as another mechanism of the anti-inflammatory activity in early phase of carrageenan-induced paw oedema.

In the present study, we have conducted four experiments to study the mechanism of action of anti-inflammatory activity produced by P.o. In addition to these specific mechanisms, several other mechanisms may account for anti-inflammatory activity of P.o. Planas et al. [42] evaluated the opioid receptor-mediated activities on suppression of the inflammation in the carrageenan-induced paw oedema. The SFDP P.o and AE of P.o have been shown to act via opioid mechanisms in inducing antinociception action in rats $[9,43]$. Therefore, P.o may also act via opioid receptors to induce anti-inflammatory activity.

Jedinak et al. [22] showed that water extract of P.o suppressed LPS-induced secretion of TNF- $\alpha$, cytokines, and activation of nuclear transcription factor $\mathrm{kB}$ which can be related to the mechanism of suppression of carrageenan-induced inflammation by SFDP and AE of P.o in the present study.

As evident by the findings of our previous study, P.o do not possibly exert any toxic effects in Wistar rats as well as human subjects [14]. Preliminary phytochemical analysis revealed the presence of terpenoids, tannins, steroidal glycosides, and carbohydrates [44].

\section{Conclusions}

The study suggests that culinary mushroom P.o possesses anti-inflammatory activity and can be considered as a functional food that has the potential to control inflammation. Antihistamine and membrane stabilizing activities, inhibition of cell migration to the site of inflammation, and inhibition of NO production may be possible mechanisms of anti-inflammatory activity of P.o. Anti-inflammatory activity-guided fractionation of $\mathrm{AE}$ of $P . o$ in the search of potent anti-inflammatory compounds is in progress. We recommend that the potential health benefits of the oyster mushroom be evaluated with clinical studies.

\section{Data Availability}

The data used to support the findings of this study are included within the article.

\section{Disclosure}

Preliminary results of the bioassay conducted using carrageenan-induced paw oedema model in healthy Wistar rats were presented at the $18^{\text {th }}$ Congress of the International Society for Mushroom Science, China.

\section{Conflicts of Interest}

The authors declare that they have no conflicts of interest.

\section{Acknowledgments}

The authors acknowledge the staff of the Animal House, Faculty of Medical Sciences, University of Sri Jayewardenepura, Sri Lanka, for providing the necessary facilities to carry out the study. Financial assistance by the University of Sri Jayewardenepura, Sri Lanka, is gratefully acknowledged (Grant nos.: ASP/06/Re/2008/04 and Ph.D./04/2012).

\section{References}

[1] M. J. Mycek, R. A. Harvvey, and P. C. Champe, "Anti-inflammatory drugs and autacoids," in Lippincott's Illustrated Reviews: Pharmacology, R. Harvvey and P. C. Champe, Eds., p. 401, Lippincott Williams and Wilkins, Pennsylvania, PA, USA, 2001.

[2] H. P. Rang, M. M. Dale, J. M. Ritter et al., Rang and Dale's Pharmacology, Churchill Livingstone, London, UK, 7th edition, 2012.

[3] L. Sautebin, "Prostaglandins and nitric oxide as molecular targets for anti-inflammatory therapy," Fitoterapia, vol. 71, no. 1, pp. S48-S57, 2000.

[4] N. Mattison, A. Gene Trimble, and L. Lasagna, "New drug development in the United States, 1963 through 1984," Clinical Pharmacology and Therapeutics, vol. 43, no. 3, pp. 290-301, 1988.

[5] S. R. Suseem and S. A. Mary, "Studies on anti-inflammatory and antipyretic activities of fruiting bodies of Pleurotus eous in experimental animals," Pharmacologyonline, vol. 1, pp. 721727, 2011.

[6] N. Jose, T. A. Ajith, and K. K. Janardhanan, "Antioxidant, anti-inflammatory, and antitumor activities of culinary-medicinal mushroom Pleurotus pulmonarius (Fr). Quel. (Agaricomycetideae)," International Journal of Medicinal Mushrooms, vol. 4, no. 4, pp. 60-67, 2002.

[7] M. Arawwawala, I. Thabrew, L. Arambewela, and S. Handunnetti, "Anti-inflammatory activity of Trichosanthes cucumerina Linn. in rats," Journal of Ethnopharmacology, vol. 131, no. 3, pp. 538-543, 2010.

[8] S. Handunnetti, Z. Bano, and P. G. Miles, "Pleurotus mushrooms. Part I A. morphology, life cycle, taxonomy, breeding, and cultivation," CRC Critical Reviews in Food Science and Nutrition, vol. 26, no. 2, pp. 157-223, 1987.

[9] N. S. Vasudewa, D. T. U. Abeytunga, and W. D. Ratnasooriya, "Antinociceptive activity of Pleurotus ostreatus., an edible mushroom, in rats," Pharmaceutical Biology, vol. 45, no. 7, pp. 533-540, 2007.

[10] P. Bobek, L. Ozdin, and S. Galbavy, "Dose- and time-dependent hypocholesterolemic effect of oyster mushroom (Pleurotus ostreatus) in rats," Nutrition, vol. 14, no. 3, pp. 282-286, 1998.

[11] D. P. Dissanayake, D. T. U. Abeytunga, N. S. Vaudewa et al., "Inhibition of lipid peroxidation by extracts of Pleurotus ostreatus," Pharmacognosy Magazine, vol. 5, no. 19, pp. 266-271, 2009. 
[12] I. Sarangi, D. Ghosh, S. K. Bhutia, S. K. Mallick, and T. K. Mallick, "Anti-tumor and immunomodulating effects of Pleurotus ostreatus mycelia-derived proteoglycans," International Immunopharmacology, vol. 6, no. 8, pp. 1287-1297, 2006.

[13] W. J. A. B. N. Jayauriya, T. S. Suresh, D. T. U. Abeytunga et al., "Oral hypoglycaemic activity of culinary-medicinal mushrooms Pleurotus ostreatus and P. cystidiosus (higher basidiomycetes) in normal and alloxan-induced diabetic wistar rats," International Journal of Medicinal Mushrooms, vol. 14, no. 4, pp. 347-55, 2012.

[14] W. J. A. B. N. Jayasuriya, C. A. Wanigatunge, G. H. Fernando, D. T. U. Abeytunga, and T. S. Suresh, "Hypoglycaemic activity of culinary Pleurotus ostreatus and P.cystidiosus mushrooms in healthy volunteers and type 2 diabetic patients on diet control and the possible mechanisms of action," Phytotherapy Research, vol. 29, no. 2, pp. 303-309, 2015.

[15] D. T. U. Abeytunga, "Biological activities of Pleurotus mushrooms," in Mushrooms: Types, Properties and Nutrition, S. Andres and N. Baumann, Eds., pp. 329-50, Nova Science Publishers, Hauppauge, NY, USA, 2011.

[16] P. Pinal, P. Natvarlal, P. Dharmik et al., "In vivo evaluation of Pleurotus sajorcaju mycelium extract for anti-inflammatory activity," Pharmacologyonline, vol. 2, pp. 784-789, 2011.

[17] N. Jose, T. A. Ajith, and K. K. Janardhanan, "Methanol extract of the oyster mushroom, Pleurotus Florida, inhibits inflammation and platelet aggregation," Phytotherapy Research, vol. 18, no. 1, pp. 43-46, 2004.

[18] H. Nozaki, S. Itonori, M. Sugita et al., "Mushroom acidic glycosphingolipid induction of cytokine secretion from murine T cells and proliferation of NK1.1 $\alpha / \beta$ TCR-double positive cells in vitro," Biochemical and Biophysical Research Communications, vol. 373, no. 3, pp. 435-439, 2008.

[19] J.-N. Chen, E. G. de Mejia, and J. S.-B. Wu, "Inhibitory effect of a glycoprotein isolated from Golden Oyster mushroom (Pleurotus citrinopileatus) on the lipopolysaccharide-induced inflammatory reaction in RAW 264.7 macrophage," Journal of Agricultural and Food Chemistry, vol. 59, no. 13, pp. 70927097, 2011.

[20] V. Bobek and C. Galbavy, "Effects of pleuran ( $\beta$-glucan isolated from Pleurotus ostreatus) on experimental colitis in rats," Physiological Research, vol. 50, no. 6, pp. 575-81, 2001.

[21] N. Rivero-Pérez, M. Ayala-Martínez, A. Zepeda-Bastida et al., "Anti-inflammatory effect of aqueous extracts of spent Pleurotus ostreatus substrates in mouse ears treated with 12O-tetradecanoylphorbol-13-acetate," Indian Journal of pharmacology, vol. 48, no. 2, pp. 141-144, 2016.

[22] A. Jedinak, S. Dudhgaonkar, Q. Wu et al., "Anti-inflammatory activity of edible oyster mushroom is mediated through the inhibition of NFkB and AP-1 signaling," Nutrition Journal, vol. 10, no. 1, p. 52, 2011.

[23] C. A. Winter, E. A. Risley, and C. W. Nuss, "Carrageenaninduced oedema in hind paws of the rat as an assay for antiinflammatory drugs," Proceedings of the Society for Experimental Biology and Medicine, vol. 111, pp. 5444-547, 1962.

[24] S. M. Handunnetti, R. R. Kumara, S. A. Deraniyagala et al., "Anti-inflammatory activity of Ixora coccinea methanolic leaf extract,” Pharmacognosy Research, vol. 1, no. 2, pp. 80-90, 2009.

[25] M. Maccioni, L. E. Cabezas, and V. E. Rivero, "Effect of prostatein, the major protein produced by the rat ventral prostate, on phagocytic cell functions," American Journal of Reproductive Immunology, vol. 50, no. 6, pp. 473-480, 2003.
[26] B. Radhika and K. Shravani, "In vitro and in vivo anti-inflammatory activity of Bauhinia X Blackeana Linn leaves," Journal of Medical Biology Science, vol. 1, no. 1, p. 102, 2018.

[27] A. Oyekachukwu, J. Elijah, O. Eshu et al., "Anti-inflammatory effects of the chloroform extract of Annona muricata leaves on phospholipase A2 and prostaglandin synthase activities," Translational Biomedicine, vol. 8, p. 137, 2017.

[28] R. Vinegar, W. Schreiber, and R. Hugo, "Biphasic development of carrageenin oedema in rats," The Journal of Pharmacology and Experimental Therapeutics, vol. 166, no. 1, pp. 96-103, 1969.

[29] N. Raval, B. Ravishankar, and B. Ashok, "Anti-inflammatory effect of Chandrashura (Lepidium sativum Linn.) an experimental study," AYU (An International Quarterly Journal of Research in Ayurveda), vol. 34, no. 3, pp. 302-304, 2013.

[30] S. V. G. Vigil, R. de Liz, Y. S. Medeiros, and T. S. Fröde, "Efficacy of tacrolimus in inhibiting inflammation caused by carrageenan in a murine model of air pouch," Transplant Immunology, vol. 19, no. 1, pp. 25-29, 2008.

[31] J. I. Fröde, L. Abraham, S. R. Heckbert et al., "The relation of markers of inflammation to the development of glucose disorders in the elderly: the cardiovascular health study," Diabetes, vol. 50, no. 10, pp. 2384-2389, 2001.

[32] M. J. Husain, M. Peakman, H. Gallati et al., "Elevated serum levels of macrophage-derived cytokines precede and accompany the onset of IDDM," Diabetologia, vol. 39, no. 1, pp. 60-69, 1996.

[33] R. Davis and N. Maro, "Aloe vera and gibberellin. Anti-inflammatory activity in diabetes," Journal of the American Podiatric Medical Association, vol. 79, no. 1, pp. 24-26, 1989.

[34] Z. Hassanabad, Z. Gholamnezhad, M. Jafarzadeh et al., "The anti-inflammatory effects of aqueous extract of ginger root in diabetic mice," Daru, vol. 13, no. 2, pp. 70-73, 2005.

[35] J. R. Vane, Y. S. Bakhle, and R. M. Botting, "Cyclooxygenases 1 and 2," Annual Review of Pharmacology and Toxicology, vol. 38, no. 1, pp. 97-120, 1998.

[36] Y. Zhang, G. L. Mills, and M. G. Nair, "Cyclooxygenase inhibitory and antioxidant compounds from the mycelia of the edible mushroom Grifola frondosa," Journal of Agricultural and Food Chemistry, vol. 50, no. 26, pp. 7581-7585, 2002.

[37] V. P. Nacife, M. d. N. C. Soeiro, R. N. Gomes, H. D'Avila, H. C. C.-F. Neto, and M. d. N. L. Meirelles, "Morphological and biochemical characterization of macrophages activated by carrageenan and lipopolysaccharide in vivo," Cell Structure and Function, vol. 29, no. 2, pp. 27-34, 2004.

[38] J. L. Wallace, "Nitric oxide as a regulator of inflammatory processes," Memórias do Instituto Oswaldo Cruz, vol. 100, no. 1, pp. 5-9, 2005.

[39] K. Kim, Y. G. Kwon, H. T. Chung et al., "Methanol extract of Cordyceps pruinosa inhibits in vitro and in vivo inflammatory mediators by suppressing NF- $\mathrm{kB}$ activation," Toxicology and Applied Pharmacology, vol. 190, no. 1, pp. 1-8, 2003.

[40] C. A. Anosike, O. Obidoa, and L. U. S. Ezeanyika, "Membrane stabilization as a mechanism of the anti-inflammatory activity of methanol extract of garden egg (Solanum aethiopicum)," DARU Journal of Pharmaceutical Sciences, vol. 20, no. 1, pp. 76-82, 2012.

[41] S. Umukoro and R. B. Ahorobi, "Evaluation of anti-inflammatory and membrane stabilizing property of aqueous leaf extract of Momordica charantia in rats," African Journal of Biomedical Research, vol. 9, no. 2, pp. 119-124, 2006.

[42] E. Planas, S. Sánchez, L. Rodriguez, O. Pol, and M. M. Puig, "Antinociceptive/anti-edema effects of liposomal morphine 
during acute inflammation of the rat paw," Pharmacology, vol. 60 , no. 3, pp. 121-127, 2000.

[43] N. S. Vasudewa, D. T. U. Abeytunga, and W. D. Ratnasooriya, "Activity-directed fractionation of Pleurotus ostreatus. In the search for analgesics," Pharmaceutical Biology, vol. 46, no. 5, pp. 295-301, 2008.

[44] B. A. Iwalokun, A. U. Usen, A. A. Otunbaau, and K. D. Olukoya, "Phytochemical evaluation, antimicrobial and antioxidant properties of Pleurotus ostreatus," African Journal of Biotechnology, vol. 6, no. 15, pp. 1732-1739, 2007. 